\title{
A New Method for Biostatistical miRNA Pattern Recognition with Topological Properties of Visibility Graphs in 3D Space
}

\author{
Matej Babič ${ }^{D},{ }^{1}$ Ninoslav Marina, ${ }^{2}$ Andrej Mrvar, ${ }^{3}$ Kumar Dookhitram ${ }^{(D)}{ }^{4}$ \\ and Michele Calì $\mathbb{D D}^{5}$ \\ ${ }^{1}$ Faculty of Information Studies, Novo Mesto, Slovenia \\ ${ }^{2}$ University of Information Science and Technology "St Paul the Apostle", Skopje, North Macedonia \\ ${ }^{3}$ Faculty of Social Sciences, University of Ljubljana, Ljubljana, Slovenia \\ ${ }^{4}$ University of Technology, Mauritius, Port Louis, Mauritius \\ ${ }^{5}$ Electric, Electronics and Computer Engineering Department, University of Catania, Catania, Italy \\ Correspondence should be addressed to Matej Babič; babicster@gmail.com
}

Received 21 December 2018; Revised 2 April 2019; Accepted 12 May 2019; Published 11 June 2019

Academic Editor: Jingfeng Jiang

Copyright (C) 2019 Matej Babič et al. This is an open access article distributed under the Creative Commons Attribution License, which permits unrestricted use, distribution, and reproduction in any medium, provided the original work is properly cited.

\begin{abstract}
Visibility is a very important topic in computer graphics and especially in calculations of global illumination. Visibility determination, the process of deciding which surface can be seen from a certain point, has also problematic applications in biomedical engineering. The problem of visibility computation with mathematical tools can be presented as a visibility network. Instead of utilizing a 2D visibility network or graphs whose construction is well known, in this paper, a new method for the construction of 3D visibility graphs will be proposed. Drawing graphs as nodes connected by links in a 3D space is visually compelling but computationally difficult. Thus, the construction of $3 \mathrm{D}$ visibility graphs is highly complex and requires professional computers or supercomputers. A new method for optimizing the algorithm visibility network in a 3D space and a new method for quantifying the complexity of a network in DNA pattern recognition in biomedical engineering have been developed. Statistical methods have been used to calculate the topological properties of a visibility graph in pattern recognition. A new $n$ hyper hybrid method is also used for combining an intelligent neural network system for DNA pattern recognition with the topological properties of visibility networks of a 3D space and for evaluating its prospective use in the prediction of cancer.
\end{abstract}

\section{Introduction}

Manufacturing the visibility network (graph) [1] is a fundamental geometric structure which has useful applications in several fields including illumination and rendering, motion planning, pattern recognition, and sensor networks. A graph $G$ is called a visibility graph when there is a polygon $P$ such that the vertices of $P$ are the vertices of $G$ and two vertices are adjacent in $G$ if they are visible in $P$. A visibility graph can be used in spatial analysis of urban and building spaces and applied to landscapes, as well. It is formed by taking a set of points across the space and forming graph edges between those points, if they are mutually visible. Visibility graphs have been widely used for 2D applications so far, but in this paper, an application to complex $3 \mathrm{D}$ visibility problems is advanced.
Visibility calculations are central to any computer graphics application. The relevance of statistics [2] has been much more recognized in the biomedical engineering field. Statistics can provide technicians of laboratories with important instruments for a scientific analysis of biomedical phenomena, allowing to understand the observed phenomena more correctly and to obtain more reliable results.

Biomedical engineering [3] being one of the fastest growing engineering disciplines aims at applying engineering expertise and advances to the field of medical needs and bioscience for the enhancement of healthcare.

Deoxyribonucleic acid (DNA) pattern recognition constitutes one of the most important works in biomedical engineering. Pattern recognition [4] is a branch of machine learning that focuses on the recognition of patterns and regularities in data, although it is in considered to be in some 
cases nearly synonymous with machine learning. In pattern recognition, labels are assigned to objects and all objects are described by features, also called attributes. A classic example is the recognition of handwritten digits for the purpose of automatic mail sorting. Pattern recognition methods based on machine learning techniques [5] have been shown to be a promising approach to the analysis of network data. Intelligent computing has attracted many scientists and researchers working on intelligent techniques for solving complex real-world problems.

The graphical representations of the DNA as a method for DNA pattern recognition have been introduced to facilitate comparison of DNA sequences and to observe differences in their structure [6-10]. A novel method is thus explored in this study to generate and characterize complex networks by means of analysis of their miRNA sequences.

A new hybrid method [11] that combines three types of intelligent neural network systems [12] has been used. This paper explores the use of an intelligent system [13] with a new hybrid method that improves the existing ones. It is based on the $n$-hyper hybrid method. The aim of this work is to outline possibilities for applying an $n$-hyper hybrid system method for miRNA pattern recognition [14] with topological properties of visibility graphs in a 3D space and to evaluate its prospective use in biomedical engineering.

The proposed method can be utilized to approach more systematically problems of visibility of miRNA sequences, in the comparison of DNA sequences and in the analysis of complex networks such as miRNA networks in the biomedical field. The method is based on an algorithm which is built for optimizing the visibility in a 3D space and an $n$ hyper hybrid system for miRNA cancer pattern recognition which allows to register more precisely the risk of various types of cancer.

In particular, the proposed method can be used to analyze the transformation of $1 \mathrm{D}$ miRNA sequences into 3D miRNA sequences for predicting cancer. More specifically, a new type of intelligent system, the $n$-hyper hybrid system, has been developed to describe miRNA sequences and the difference between cancer and noncancer miRNA. Thanks to this method, some more information on a complex network derived from the visibility network in a 3D space, compared to the visibility graph, can be obtained in the application of miRNA pattern recognition. The utility of applying $3 \mathrm{D}$ visibility graphs in biomedical engineering for a more precise prediction of cancer will be thus discussed in this paper.

Finally, this new method permits to obtain a physical visualization of the three-dimensional miRNA sequences. Through additively manufactured (3D printing) techniques, which are much used in the biomedical field for building and analysis of implantable devices [15-18], it is possible indeed to have a reconstruction of the $3 \mathrm{D}$ space network as a lattice structure as well as a view of the relationships between miRNA sequences.

This paper is organized as follows: Section 2 is devoted to a description of the methods and the materials used. In Section 3, the results and discussions are illustrated. Final considerations and conclusions are drawn in Section 4.

\section{Materials and Methods}

2.1. A New Method for Statistical miRNA Pattern Recognition. DNA [19-23] is composed of an extremely long array of nucleotides. MicroRNAs [23-27] constitute a recently discovered class of noncoding RNAs playing some key roles in the regulation of gene expression. In this article, we have developed a new method to describe the transformation of $1 \mathrm{D}$ miRNA sequences into $3 \mathrm{D}$ miRNA sequences. This means that we have transformed the miRNA sequences into a 3D coordinate system.

For better presentation, four colours are used to denote the nucleotides adenine $(A)$, cytosine $(C)$, guanine $(G)$, and thymine $(\mathrm{T})$. We have replaced each nucleotide by different colours, namely, $\mathrm{A}$ by white, $\mathrm{C}$ by light purple, $\mathrm{G}$ by gray, and $\mathrm{T}$ by black. Initially, we have transformed each nucleotide of the $D N A$ sequences into a $2 \mathrm{D}$ array by using a spiral curve. Figure 1 presents the coloured miRNA sequences (miR-612 gene) and transformation of each nucleotide of the miRNA sequences into a $2 \mathrm{D}$ array by using a spiral curve. We have also denoted the miRNA sequences by two coordinates $(x, y)$.

Furthermore, we have denoted each nucleotide of the miRNA sequences with a number. An example of an miRNA sequence is ... TGCCAATCGTTGT .... This sequence of letters can be converted with the function $f$. We have denoted $\{A, C, G, T\} \in M$ and $\{1,2,3,4\} \in N$. Also, $f$ : $M \longrightarrow N$. We have denoted with $n_{\mathrm{A}}, n_{\mathrm{C}}, n_{\mathrm{G}}$, and $n_{\mathrm{T}}$ the number of all nucleotides $A, C, G$, and $T$ in some sequences. We have determined linear arrangement $\left\{n_{\mathrm{A}}, n_{\mathrm{C}}, n_{\mathrm{G}}, n_{\mathrm{T}}\right\}$ from the largest to the smallest value. For example, if $n_{\mathrm{A}}>n_{\mathrm{C}}>n_{\mathrm{G}}>n_{\mathrm{T}}$, then we have function $f, f: A \longrightarrow 4, f$ : $C \longrightarrow 3, f: G \longrightarrow 2$, and $f: T \longrightarrow 1$ or $f(A)=4, f(C)=3, f(G)=$ 2 , and $f(T)=1$. When using function $f$, we can write $f(\ldots$ TGCCAATCGTTGT ...) = . . $1233441231121 \ldots$. . Also, the function $f$ denotes the third coordinate of the miRNA nucleotides in a 3D space, $(x, y, z)$. In the third step, we have used a new method for optimizing the algorithm visibility graph in a $3 \mathrm{D}$ space (Figure 2).

For each visibility graph of the miRNA sequences, we have calculated the statistical property chi-square of triads [28].

If $v$ independent variables $x_{i}$ are each normally distributed with mean $\mu_{i}$ and variance $\sigma_{i}^{2}$, then the quantity known as chi-square $\left(\chi^{2}\right)$ is denoted by

$$
\chi^{2}=\frac{\sum\left(x_{i}-\mu_{i}\right)^{2}}{\sigma_{i}^{2}} \text {. }
$$

2.2. A New Method for Optimizing the Algorithm Visibility Graph in 3D Space. We have developed a new algorithm for the construction of visibility graphs in a 3D space [29]. Two arbitrary data values $\left(x_{a}, y_{a}\right)$ and $\left(x_{b}, y_{b}\right)$ will have visibility and will consequently become two connected nodes of the associated graph if any other data $\left(x_{c}, y_{c}\right)$ placed between them fulfil the following relation [30]:

$$
y_{c}<y_{b}+\frac{\left(y_{a}-y_{b}\right)\left(x_{b}-x_{c}\right)}{x_{b}-x_{a}} \text {. }
$$




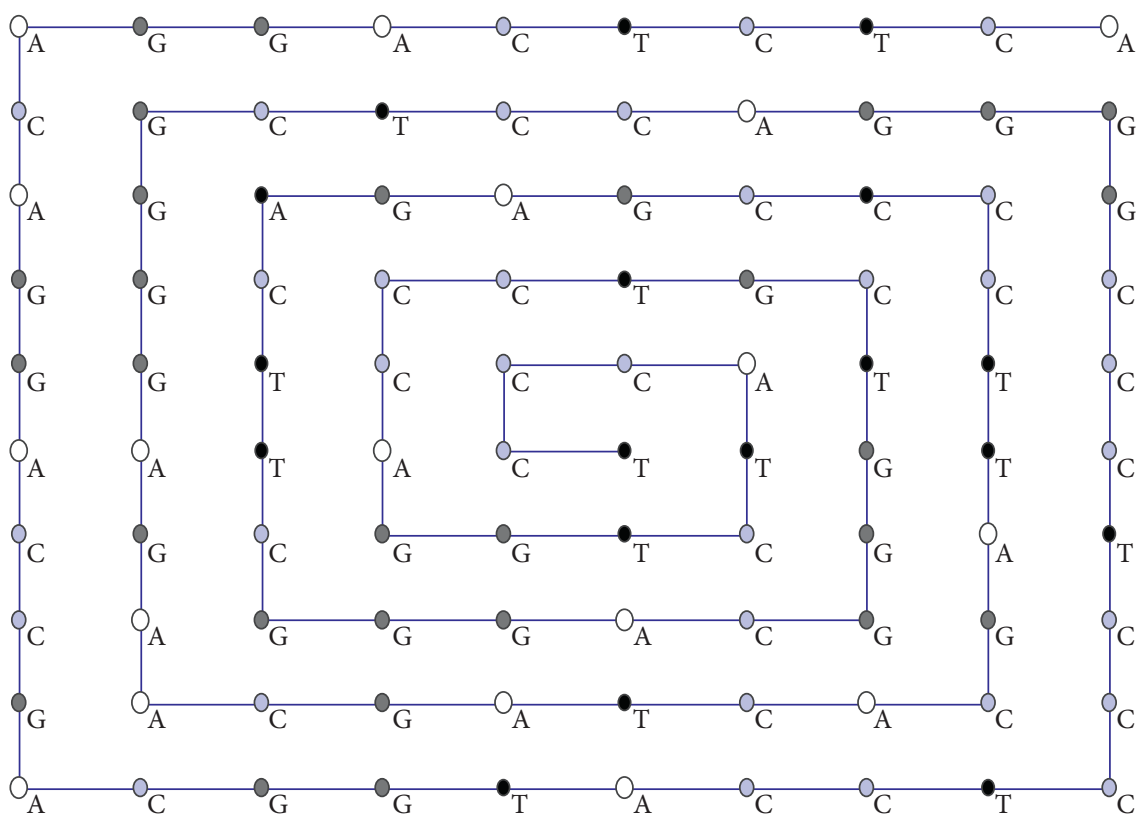

FIGURE 1: Coloured miRNA sequences (miR-612 gene) and transformation of each nucleotide of the DNA sequences into a 2D array by using a spiral curve.

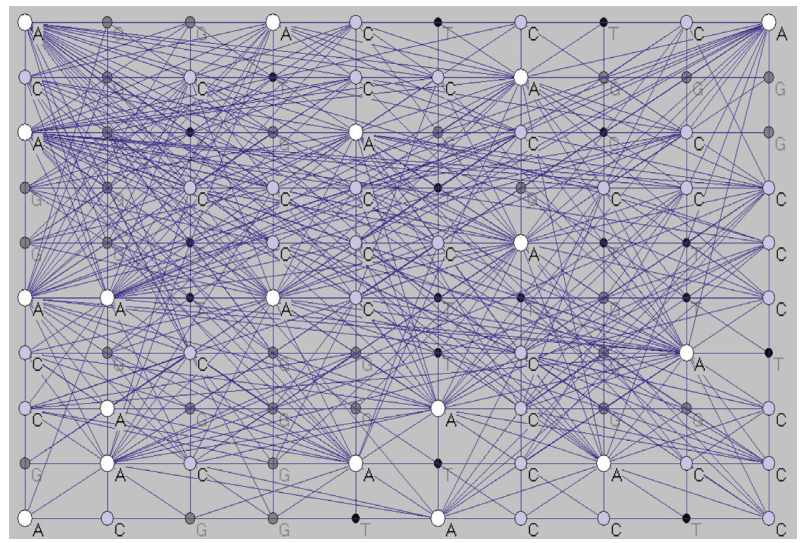

Figure 2: Visibility graph of miRNA sequences (miR-612 gene).

We wanted to know how to connect the nodes in Figure 3. Nodes $v_{i, j}$ and $v_{k, l}$ of the 3D graph, where $i<k$ and $j<l$, are connected by a link if and only if they are visible.

This means that the path from $v_{i, j}$ to $v_{k, l}$ has no points on the graph. An example is presented in Figure 3 , in which the nodnes connected by the blue line are visible to each other and those connected by the red line are an example of unrelated nodes (the straight line that connects the two nodes pierces the graph, which is contrary to the definition of the visibility graph). The following section describes how to construct a 3D visibility graph. The open problem of visibility graphs in a $2 \mathrm{D}$ space has thus been presented (Figure 4). Also, we have transformed all 3D points by perpendicular projection on the $x y$ plane.

As a first step, we have the $3 \mathrm{D}$ points which are transformed into a $2 \mathrm{D}$ plane. A graphical solution on a $5 \times 5$ grid is given because it provides a better visual representation. Figure 5 presents the nodes of the graph.

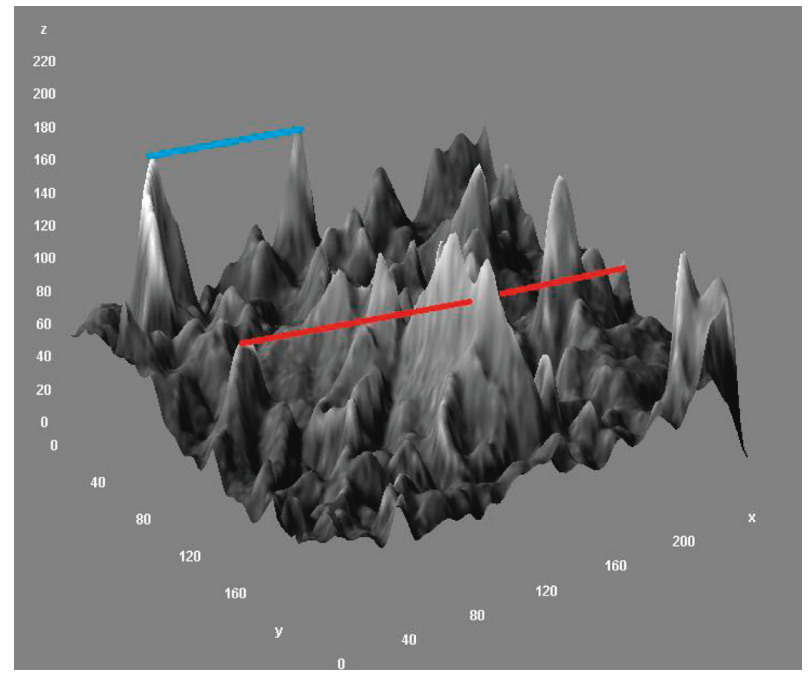

FIGURE 3: Visibility nodes (blue line) and unrelated nodes (red line).

In the second step, we have connected neighbouring nodes. Also, node $T_{i, j}\left(x_{i}, y_{j}\right)$ is connected with nodes $T\left(x_{i-1}\right.$, $\left.y_{j}\right), T\left(x_{i+1}, y_{j}\right), T\left(x_{i}, y_{j+1}\right)$, and $T\left(x_{i}, y_{j-1}\right)$ if node $T_{i, j}\left(x_{i}, y_{j}\right)$ is not located on the edge of a complex network. If node $T_{i, j}\left(x_{i}\right.$, $y_{j}$ ) is on the edge of a complex network, then it is connected with two or three nodes only, as presented in Figure 6.

In the third step, nodes $T_{i, j}\left(x_{i}, y_{j}\right), T\left(x_{i+1}, y_{j}\right), T\left(x_{i+1}, y_{j+1}\right)$, and $T\left(x_{i}, y_{j+1}, z_{i, j+1}\right)$ present quadrilaterals, and it is possible to see many quadrilaterals in Figure 7. In all quadrilaterals, nodes from a higher $z$ coordinate are connected with other nodes by diagonal lines.

In the fourth step (Figure 8), we have broken the set of all nodes $\left(x_{i}, y_{i}, z_{i, j}\right)$ into two sets. The first set presents all nodes $\left(x_{i}, 0, z_{i, j}\right)$, and the second set presents all nodes $\left(0, y_{i}, z_{i, j}\right)$. 

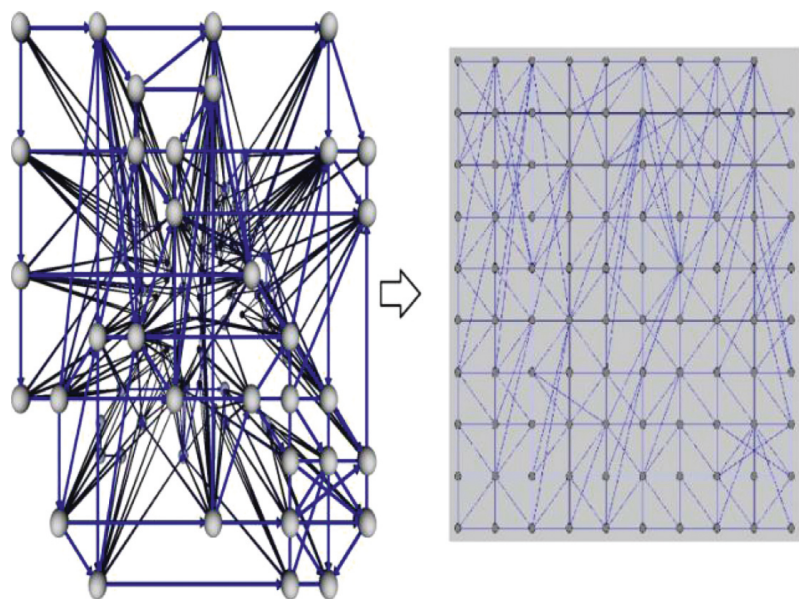

FIgURE 4: Solution of the $3 \mathrm{D}$ visibility graph presented as a $3 \mathrm{D}$ graph in a $3 \mathrm{D}$ space and in a $2 \mathrm{D}$ space.

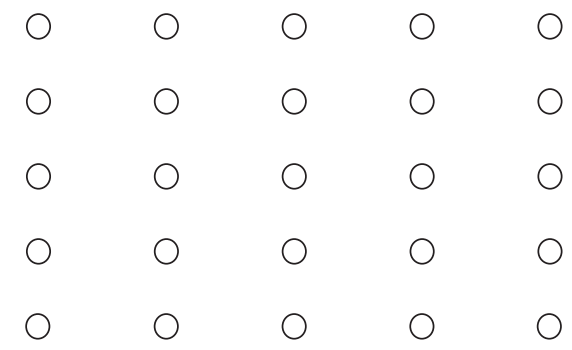

FIgURE 5: 3D nodes which are transformed into a $2 \mathrm{D}$ plane.

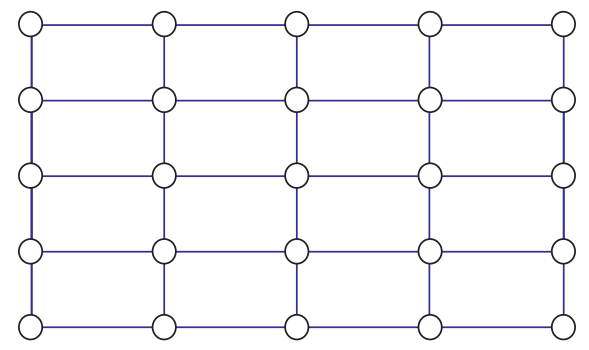

FIgURE 6: Neighbouring nodes.

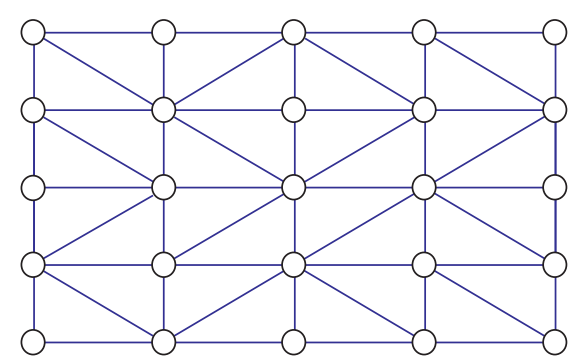

Figure 7: Nodes connected with other nodes by diagonal lines.

We have presented those nodes that are visible in the first set and those that are visible in the second set. In the first set, two nodes $T_{i, j}\left(x_{i}, y_{j}, z_{i, j}\right)$ and $T_{k, l}\left(x_{k}, y_{l}, z_{k, l}\right)$ are visible and will consequently become connected nodes on the associated

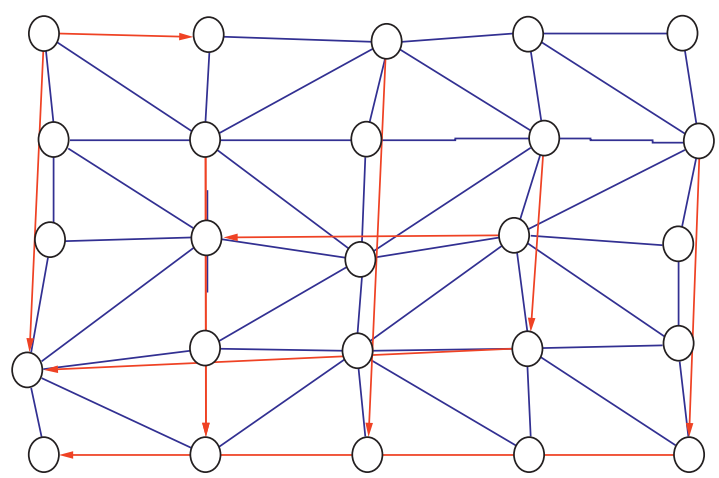

FIGURE 8: Fourth step of visibility graph creation.

graph if any other node $T\left(x_{m}, 0, z_{m, 0}\right)$ placed between them fulfils the following relation:

$$
z_{m, 0}<z_{k, l}+\frac{\left(z_{i, j}-z_{k, l}\right)\left(x_{k}-x_{m}\right)}{\left(x_{k}-x_{i}\right)} .
$$

In the second set, two nodes $T_{i, j}\left(x_{i}, y_{j}, z_{i, j}\right)$ and $T_{k, l}\left(x_{k}, y_{l}\right.$, $\left.z_{k, l}\right)$ are visible and will consequently become two connected nodes on the associated graph if any other node $T_{0, n}\left(0, y_{n}\right.$, $\left.z_{0, n}\right)$ placed between them fulfils the following relation:

$$
z_{m, 0}<z_{k, l}+\frac{\left(z_{i, j}-z_{k, l}\right)\left(y_{k}-y_{m}\right)}{\left(y_{k}-y_{i}\right)} .
$$

In the fifth step, we have connected all other visible nodes. Two nodes $T_{i, j}\left(x_{i}, y_{j}, z_{i, j}\right)$ and $T_{k, l}\left(x_{k}, y_{l}, z_{k, l}\right)$ will be visible if no nodes exist on the line between $T_{i, j}\left(x_{i}, y_{j}, z_{i, j}\right)$ and $T_{k, l}\left(x_{k}, y_{l}, z_{k, l}\right)$. Figure 9 presents the solution of the $3 \mathrm{D}$ visibility graph on a $5 \times 5$ grid.

\subsection{Optimizing the Algorithm Visibility Network in 3D Space}

(i) All $3 \mathrm{D}$ points transform into $2 \mathrm{D}$ points $\{(x, y$, $z) \longrightarrow(x, y)\}$

(ii) Node $T_{i, j}\left(x_{i}, y_{j}\right)$ is connected with nodes $T\left(x_{i-1}, y_{j}\right)$, $T\left(x_{i+1}, y_{j}\right), T\left(x_{i}, y_{j+1}\right)$, and $T\left(x_{i}, y_{j-1}\right)$, if node $T_{i, j}\left(x_{i}\right.$, $y_{j}$ ) is not located on the edge of a complex network

(iii) If node $T_{i, j}\left(x_{i}, y_{j}\right)$ is on the edge of a complex network, then it is connected with two or three nodes only

(iv) Nodes from a higher $z$ coordinate of all nodes $T_{i, j}\left(x_{i}\right.$, $\left.y_{j}\right), T\left(x_{i+1}, y_{j}\right), T\left(x_{i+1}, y_{j+1}\right)$, and $T\left(x_{i}, y_{j+1}, z_{i, j+1}\right)$ in quadrilaterals are connected with other nodes by diagonal lines

(v) Two nodes $T_{i, j}\left(x_{i}, y_{j}, z_{i, j}\right)$ and $T_{k, l}\left(x_{k}, y_{l}, z_{k, l}\right)$ are connected, if any other node $T\left(x_{m}, 0, z_{m, 0}\right)$ placed between them fulfils $z_{m, 0}<z_{k, l}+\left(z_{i, j}-z_{k, l}\right)\left(x_{k}-x_{m}\right) /$ $\left(x_{k}-x_{i}\right)$

(vi) Two nodes $T_{i, j}\left(x_{i}, y_{j}, z_{i, j}\right)$ and $T_{k, l}\left(x_{k}, y_{l}, z_{k, l}\right)$ are connected, if any other node $\mathrm{T}_{0, n}\left(0, y_{n}, z_{0, n}\right)$ placed between them fulfils $z_{m, 0}<z_{k, l}+\left(z_{i, j}-z_{k, l}\right)\left(y_{k}-y_{\mathrm{m}}\right) /$ $\left(y_{k}-y_{i}\right)$

(vii) Two nodes $T_{i, j}\left(x_{i}, y_{j}, z_{i, j}\right)$ and $T_{k, l}\left(x_{k}, y_{l}, z_{k, l}\right)$ are connected, if no nodes exist on the line between $T_{i, j}\left(x_{i}, y_{j}, z_{i, j}\right)$ and $T_{k, l}\left(x_{k}, y_{l}, z_{k, l}\right)$ 


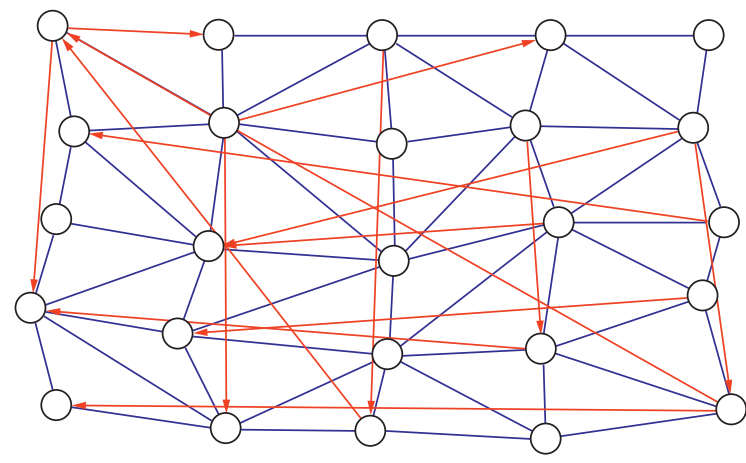

FIGURE 9: Solution of the $3 \mathrm{D}$ visibility graph on a $5 \times 5$ grid.

2.4. A New n-Hyper Hybrid Method. Hybrid evolutionary computation is a generic, flexible, robust, and versatile method for solving complex global optimization problems and can also be used in practical applications. Only three methods are adopted using intelligent systems, namely, the neural networks NN1 (present prediction with 33\%; also, we use $67 \%$ of the data for the learning set and $33 \%$ for the test set), NN2 (present prediction with $50 \%$; also, we use $50 \%$ of the data for the learning set and $50 \%$ for the test set), and NN3 (present leave-one-out cross-validation method). We have used a four-layer network with a learning rate of 0.7 , moment of learning of 0.6 , tolerance of test set of 0.02 , and tolerance of learning set of 0.2 . Hybrid 1 presents the sequence hybrid method. In this hybrid, methods are connected in series in the direction of the entrance to the method $n$. All methods work independently of the other methods. The results of input method 1 are transferred to input method 2, and the results of input method 2 are transferred to input method 3. Hybrid 2 presents the cyclic hybrid method. In Hybrid 2, methods are connected in series in the direction of the entrance to the method $n$. All methods work independently of the other methods.

The results of input method 1 are transferred to input method 2, the results of input method 2 are transferred to input method 3, and the results of input method 3 are transferred to input method 1. In Hybrid 3, all methods work independently of the other methods. The results of input method 1 are transferred to input method 2, the results of input method 2 are transferred to input method 3 , the results of input method 3 are transferred to input method 2, and the results of input method 2 are transferred to input method 1. 1-hyper hybrid methods are similar to hybrid methods. Also, we have repeated the process up to $n$, where $n \in N$. In the end, we have $n$-hyper hybrid methods. Figure 10 presents all the processes of building $n$-hyper hybrids.

\section{Results and Discussion}

The visibility graph problem itself has long been studied in computational geometry and has been applied to a variety of areas. We present a new method for describing the transformation of $1 \mathrm{D}$ miRNA sequences into 3D miRNA sequences. We combine this method with a new method for optimizing the algorithm visibility graph in a $3 \mathrm{D}$ space.
Based on the variation network, several topological properties, such as different types of triads, are calculated for natural miRNA sequences. Also, the correlations between types of triads over the variation network are obtained.

It is well known that there is an individual cancer susceptibility despite equivalent environmental exposure, likely due to polymorphisms in genes involved in carcinogenesis. Table 1 presents a list of miRNA gene polymorphisms associated with cancer. We use the miR-146a, hsa-mir-149, hsa-mir-196a-2, hsa-mir-608, and hsa-miR612 genes from the miRNA base. MiR-146a is a family of microRNA precursors found in mammals, including humans. MiR-146a is primarily involved in the regulation of inflammation and other processes that function in the innate immune system. Loss of functional miR-146a (and mir-145) could predispose an individual to suffer from chromosome $5 q$ deletion syndrome. MiR-146 has also been reported to be highly upregulated in the osteoarthritis cartilage and could be involved in its pathogenesis. An increasing body of evidence points to a possible role of microRNAs (miRNAs) in hereditary cancer syndromes $[31,32]$.

Recently, variations of the miR-146a gene have drawn increasing attention in cancer etiologies, and altered expression levels have been observed in inflammatory diseases as well as in cancers $[33,34]$. MicroRNA hsa-mir-149 is located on chromosome 2. It is an intronic miRNA and is located in sense orientation relative to its protein-coding host gene glypican 1 (GPC1). Our integrated review of miRNA-SNPs revealed that polymorphisms of hsa-mir-149 have previously been associated with increased or decreased risk of seven cancer types: renal cell carcinoma and breast, colorectal, gastric, hepatocellular, papillary, and thyroid cancers. miR149 rs71428439 predisposes its carriers to CCRCC, and miR149 rs71428439 may be a new biomarker for predicting the risk of CCRCC [35]. miR-196 appears to be a vertebrate-specific microRNA and has now been predicted or experimentally confirmed in a wide range of vertebrate species (MIPF0000031). The hairpin precursors are predicted based on base pairing and cross-species conservation-their extents are not known. Many studies demonstrated that the hsa-miR-196a2 rs11614913 SNP was significantly associated with the susceptibility of breast cancer [36-38]. MicroRNA hsa-mir-608 is located on chromosome 10. It is an intronic miRNA, located in sense orientation relative to its host gene semaphorin 4G (SEMA4G) and in antisense orientation to the mitochondrial ribosomal protein L43M (RPL43) gene. Several studies [39-46] examined the impact of miR-608 rs4919510C $>\mathrm{G}$ on the risk of various cancers, but the results were inconsistent.

Additionally, it has also been associated with increased risk of breast, nasopharyngeal, and papillary thyroid carcinomas. MicroRNA hsa-mir-612 is located on chromosome 11. It is an exonic miRNA, located in sense orientation relative to its host gene, nuclear paraspeckle assembly transcript 1 (NEAT1). Polymorphism with pre-miRNA regions has been associated with B-cell acute lymphoblastic leukemia [47-49].

Also, we have used a new method for optimizing the algorithm visibility graph in a 3D space and a new method 

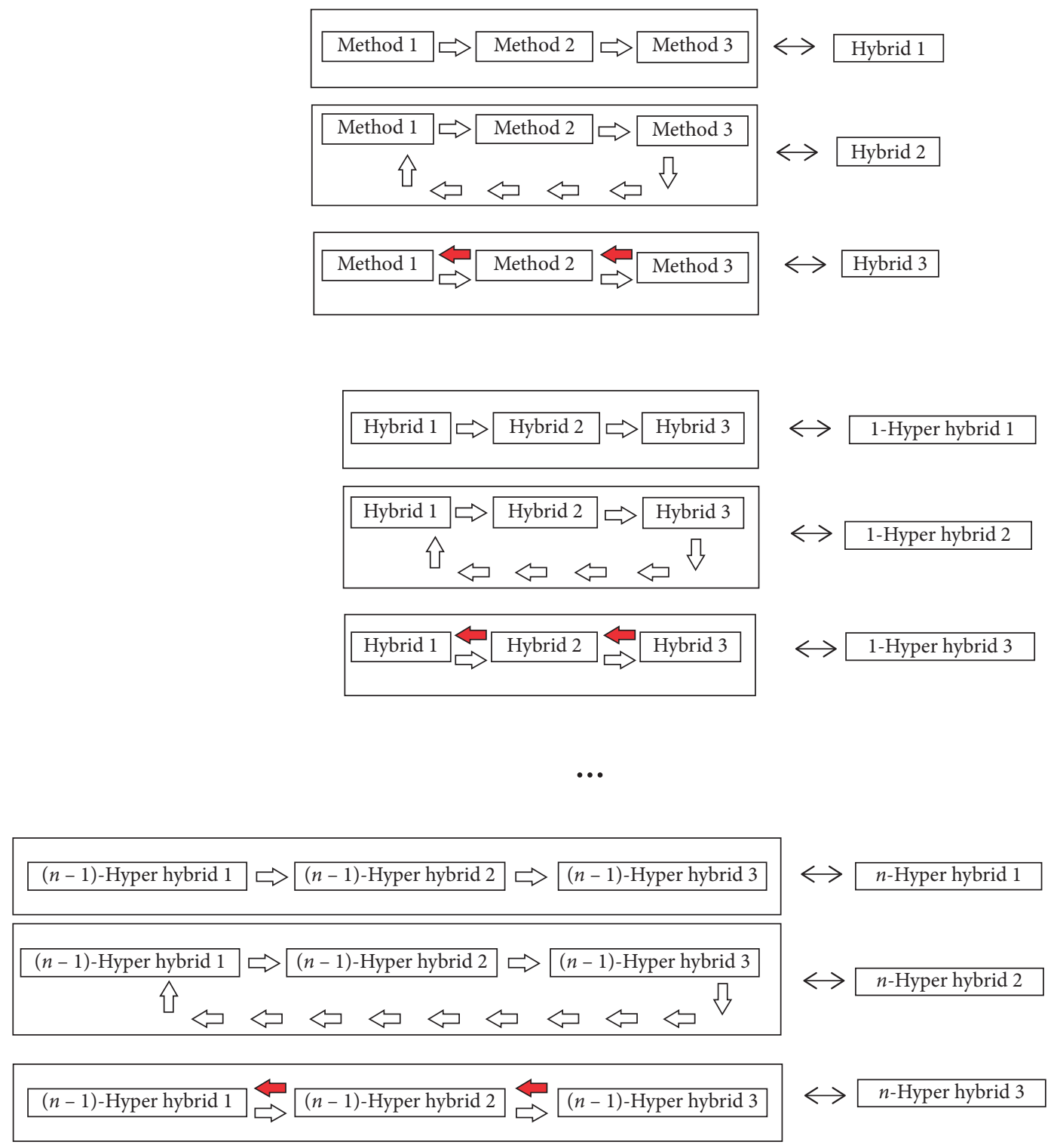

Figure 10: $n$-Hyper hybrid method.

TABLE 1: List of miRNA gene polymorphisms associated with cancer.

\begin{tabular}{|c|c|c|c|c|}
\hline miRNA name & Mark & rs number & Nucleotide change & Cancer type \\
\hline hsa-mir-146a & D1 & 2910164 & $A>G$ & Increased risk for gastric cancer \\
\hline hsa-mir-149 & D2 & 71428439 & $A>G$ & $\begin{array}{l}\text { Increased risk for chronic lymphocytic } \\
\text { leukemia }\end{array}$ \\
\hline hsa-mir-196a-2 & D3 & 11614913 & $\mathrm{C}>\mathrm{T}$ & Breast cancer \\
\hline hsa-mir-608 & D4 & 4919510 & $\mathrm{C}>\mathrm{G}$ & Increased risk for breast cancer \\
\hline hsa-mir-612 & D5 & 12803915 & $\mathrm{G}>\mathrm{A}$ & B-cell acute lymphoblastic leukemia \\
\hline
\end{tabular}

for miRNA cancer pattern recognition. We have determined the topological properties of the triad patterns, in miRNA networks [50]. The analysis of triads and the prevalence of different types of triads in populations has been a staple of most network analyses. The input data of neural networks are the topological properties, namely, the triads of miRNA patterns and function $f$ of sequences of miRNA. The output data $(Y)$ of the neural data decide whether the miRNA is associated with cancer: if the miRNA is not associated with cancer, then 1 is output, else 0 is output.

Table 2 presents the topological properties of the visibility graphs in a 3D space of DNA patterns. D1-D5 present the mark of DNA. C1-C5 present cancer D1-D5 DNA. TP1-TP10 [51] present the types of topological properties of triads: TP1 presents type 1-102, TP2 presents type 1-003, TP3 presents type 2-012, TP4 presents type 6-021C, TP5 
TABLE 2: Topological properties of the graph of miRNA patterns.

\begin{tabular}{lccccccc}
\hline$N$ & TP1 & TP2 & TP3 & TP4 & TP5 & TP6 & $Y$ \\
\hline D1 & 15359 & 126096 & 17484 & 489 & 505 & 522 & 0 \\
C1 & 15372 & 126020 & 17446 & 486 & 509 & 525 & 1 \\
D2 & 15511 & 126406 & 17240 & 497 & 514 & 469 & 0 \\
C2 & 15611 & 126482 & 17082 & 490 & 509 & 470 & 1 \\
D3 & 18875 & 167586 & 20947 & 424 & 530 & 522 & 0 \\
C3 & 18886 & 167784 & 20753 & 421 & 524 & 525 & 1 \\
D4 & 15601 & 125597 & 18093 & 402 & 488 & 480 & 0 \\
C4 & 15603 & 125596 & 18085 & 401 & 487 & 481 & 1 \\
D5 & 15611 & 126952 & 16657 & 454 & 493 & 469 & 0 \\
C5 & 15639 & 126953 & 16655 & 453 & 492 & 473 & 1
\end{tabular}

presents type 7-111D, and TP6 presents type 8-111U. For each constructed variation miRNA network, the related topological properties are shown in Table 2. We can see that triad TP1 type 1-102 increases for cancer miRNA (rows $\mathrm{C} 1-\mathrm{C} 5)$. In the third column, we can see that triad TP3 type 2-012 decreases for cancer miRNA (rows $\mathrm{C} 1-\mathrm{C} 5$ ). In the fourth column, we can see that triad TP4 type 6-021C decreases for cancer miRNA (rows $\mathrm{C} 1-\mathrm{C} 5$ ). In the fifth column, we can see that triad TP6 type 8-111U decreases for cancer miRNA (rows C1-C5). Also, these three types of triads present a significant correlation between $m i R N A$ and cancer miRNA.

Table 3 presents the statistical properties of the graph of miRNA patterns. $X$ presents the modification of the nucleotide position. $S 1$ presents the place number of changed nucleotides in miRNA. S2 presents the number of all edges in the graph. S3 presents the chi-square of triads. In hsa-mir$146 \mathrm{a}$, nucleotide A changes into $\mathrm{G}$ on the 50th place, which is described in column S1. The chi-square of the triads increases on hsa-mir-149, hsa-mir-196a-2, and hsa-mir-608 in another decrease.

Table 4 presents the experimental and predicted cancers of miRNA patterns. Column $\mathrm{C}$ presents the decision of cancer miRNA with different methods. In Table 4, rows NN1, NN2, and NN3 present the data predicted with the neural network, rows $\mathrm{H} 1, \mathrm{H} 2$, and $\mathrm{H} 3$ present the data predicted with the hybrid system, 1-H1, 1- H2, and 1-H3 present the data predicted with the 1-hyper hybrid system, 2$\mathrm{H} 1,2-\mathrm{H} 2$, and $2-\mathrm{H} 3$ present the data predicted with the 2hyper hybrid system, and $10-\mathrm{H} 1,10-\mathrm{H} 2$, and $10-\mathrm{H} 3$ present the data predicted with the 10-hyper hybrid system.

The first row is equal to column $Y$ in Table 2, which determines whether the miRNA is cancer or noncancer miRNA. Modeling with NN1 presents a $30 \%$ precision from the set of measured data, modeling with NN2 presents a $60 \%$ precision from the set of measured data, modeling with NN3 presents a $70 \%$ precision from the set of measured data, modeling with $\mathrm{H} 1$ presents a $30 \%$ precision from the set of measured data, modeling with $\mathrm{H} 2$ presents a $30 \%$ precision from the set of measured data, modeling with $\mathrm{H} 3$ presents a $30 \%$ precision from the set of measured data, modeling with $1-\mathrm{HH} 1$ presents a $30 \%$ precision from the set of measured data, modeling with $1-\mathrm{HH} 2$ presents a $50 \%$ precision from the set of measured data, modeling with $1-\mathrm{HH} 3$ presents a $60 \%$ precision from the set of measured data, modeling with $2-\mathrm{HH} 1$ presents a $30 \%$ precision from the set of measured
TABLE 3: Statistical properties of the graph of miRNA patterns.

\begin{tabular}{llllc}
\hline$N$ & $X$ & S1 & S2 & S3 \\
\hline D1 & A & 50 & 395 & 251228 \\
C1 & G & 50 & 396 & 249883 \\
D2 & A & 83 & 392 & 265112 \\
C2 & G & 83 & 390 & 269117 \\
D3 & C & 78 & 425 & 365987 \\
C3 & T & 78 & 423 & 368945 \\
D4 & C & 37 & 399 & 265911 \\
C4 & G & 37 & 399 & 266532 \\
D5 & G & 51 & 386 & 273025 \\
C5 & A & 51 & 386 & 272976 \\
\hline
\end{tabular}

TABLE 4: Experimental and predicted cancers of miRNA patterns.

\begin{tabular}{lcccccccccc}
\hline$N$ & D1 & C1 & D2 & C2 & D3 & C3 & C4 & D4 & C5 & D5 \\
\hline E & 0 & 1 & 0 & 1 & 0 & 1 & 0 & 1 & 0 & 1 \\
NN1 & 1 & 0 & 0 & 0 & 0 & 1 & 1 & 0 & 1 & 0 \\
NN2 & 1 & 1 & 1 & 1 & 0 & 0 & 0 & 1 & 1 & 1 \\
NN3 & 0 & 1 & 0 & 1 & 0 & 0 & 0 & 1 & 1 & 1 \\
H1 & 0 & 0 & 1 & 0 & 1 & 1 & 1 & 0 & 1 & 0 \\
H2 & 1 & 1 & 1 & 1 & 1 & 1 & 0 & 1 & 1 & 0 \\
H3 & 0 & 1 & 0 & 0 & 0 & 0 & 1 & 1 & 1 & 1 \\
$1-H H 1$ & 1 & 0 & 1 & 0 & 1 & 1 & 1 & 0 & 0 & 0 \\
$1-H H 2$ & 0 & 1 & 1 & 0 & 0 & 0 & 0 & 1 & 1 & 0 \\
$1-H H 3$ & 1 & 1 & 0 & 1 & 0 & 0 & 0 & 0 & 0 & 1 \\
$2-H H 1$ & 1 & 0 & 0 & 0 & 1 & 1 & 1 & 1 & 1 & 0 \\
$2-H H 2$ & 1 & 1 & 1 & 1 & 1 & 1 & 1 & 0 & 0 & 1 \\
$2-H H 3$ & 0 & 1 & 0 & 0 & 0 & 0 & 0 & 1 & 0 & 1 \\
$10-H H 1$ & 1 & 0 & 0 & 1 & 1 & 1 & 1 & 1 & 1 & 1 \\
$10-H H 2$ & 1 & 0 & 0 & 1 & 0 & 0 & 0 & 1 & 0 & 1 \\
$10-H H 3$ & 1 & 1 & 0 & 1 & 0 & 1 & 0 & 1 & 0 & 1 \\
\hline
\end{tabular}

data, modeling with $2-\mathrm{HH} 2$ presents a $50 \%$ precision from the set of measured data, modeling with $2-\mathrm{HH} 3$ presents a $70 \%$ precision from the set of measured data, modeling with $10-\mathrm{HH} 1$ presents a $50 \%$ precision from the set of measured data, modeling with $10-\mathrm{HH} 2$ presents a $70 \%$ precision from the set of measured data, and modeling with $10-\mathrm{HH} 3$ presents a $90 \%$ precision from the set of measured data. Therefore, we can see that $10-\mathrm{HH} 3$ presents the best prediction.

\section{Conclusions}

In this paper, a novel concept entitled "optimizing the algorithm visibility graph in a 3D space network" is introduced to analyze the relationships between miRNA sequences and the type of cancer miRNA. We have developed a new method for describing the transformation of $1 \mathrm{D}$ miRNA sequences into $3 \mathrm{D}$ miRNA sequences. Using the topological properties of different types of triads, we have determined miRNA sequences and the difference between cancer and noncancer miRNA. From the results obtained, we are able to conclude that the variation network is a complex network and that it has some dynamic information for further researches. The visibility network in a 3D space, which contains more information than the visibility graph, has been used for the application of miRNA pattern 
recognition in biomedical engineering. This new method permits to obtain also a physical visualization of the tridimensional miRNA sequences through additively manufactured techniques.

Finally, we have built a new type of intelligent system, the $n$-hyper hybrid system, that can be used for cancer miRNA prediction.

\section{Data Availability}

The data in Table 1 are available online at http://www.ncbi. nlm.nih.gov/pmc/articles/PMC4586796/.

\section{Conflicts of Interest}

The authors declare that there are no conflicts of interest regarding the publication of this paper.

\section{Acknowledgments}

This research was supported in part by European Union, European Social Fund, and a benefit obtained with the fund for the athenaeum research in Catania, research programme 2019/2021.

\section{References}

[1] Ben-Moshe, O. Hall-Holt, M. J. Katz, and J. S. B. Mitchell, "Computing the visibility graph of points within a polygon," in Proceedings of the 20th ACM Symposium on Computational Geometry, pp. 27-35, Brooklyn, NY, USA, June 2004.

[2] D. A. Freedman, Statistical Models: Theory and Practice, Cambridge University Press, Cambridge, UK, 2005, ISBN 978-0-521-67105-7.

[3] J. D. Enderle and J. D. Bronzino, Introduction to Biomedical Engineering, p. 16, Academic Press, Cambridge, MA, USA, 2012, ISBN 978-0-12-374979-6.

[4] N. Easterbrook, "Alternate presents: the ambivalent historicism of pattern recognition," Science Fiction Studies, vol. 33, no. 100, pp. 483-504, 2006, ISSN 0091-7729.

[5] Y. Wernick, Y. Brankov, and S. Strother, "Machine learning in medical imaging," IEEE Signal Processing Magazine, vol. 27, no. 4 , pp. 25-38, 2010.

[6] E. Hamori, "Graphical representation of long DNA sequences by methods of H curves, current results and future aspects," Biotechniques, vol. 7, pp. 710-720, 1989.

[7] B. Liao and T. Wang, "3-D graphical representation of DNA sequences and their numerical characterization," Journal of Molecular Structure: THEOCHEM, vol. 681, pp. 209-212, 2004.

[8] A. Nandy, "A new graphical representation and analysis of DNA sequence structure," Current Science, vol. 66, pp. 309314, 1994.

[9] M. Randić, M. Vračko, N. Lerš, and D. Plavšić, "Novel 2-D graphical representation of DNA sequences and their numerical characterization," Chemical Physics Letters, vol. 368, pp. 1-6, 2003.

[10] M. Randić, M. Vračko, J. Zupan, and M. Novič, "Compact 2-D graphical representation of DNA," Chemical Physics Letters, vol. 373, pp. 558-562, 2003.

[11] V. Ravi, N. Naveen, and M. Pandey, "Hybrid classification and regression models via particle swarm optimization auto associative neural network based nonlinear PCA," International Journal of Hybrid Intelligent Systems, vol. 10, no. 3, 2013.

[12] M. El Hafnawi and M. Mysara, Recurrent Neural Networks and Soft Computing, Intech, Rijeka, Croatia, 2012.

[13] A. Jameson and J. Riedl, "Introduction to the transactions on interactive intelligent systems," ACM Transactions on Interactive Intelligent Systems, vol. 1, no. 1, pp. 1-6, 2011.

[14] P. Gagniuc, P. D. Cristea, R. Tuduce, C. Ionescu-Tîrgovişte, and L. Gavrila, "DNA patterns and evolutionary signatures obtained through Kappa Index of Coincidence," Revue Roumaine Des Sciences Techniques Série Électrotechnique et Énergétique, vol. 57, no. 1, pp. 100-109, 2012.

[15] E. M. Zanetti, A. Aldieri, M. Terzini, M. Calì, G. Franceschini, and C. Bignardi, "Additively manufactured custom loadbearing implantable devices: grounds for caution," Australasian Medical Journal, vol. 10, no. 8, pp. 694-700, 2017.

[16] D. Speranza, D. Citro, F. Padula et al., "Additive manufacturing techniques for the reconstruction of 3D fetal faces," Applied Bionics and Biomechanics, vol. 2017, Article ID 9701762, 10 pages, 2017.

[17] J. De Krijger, C. Rans, B. Van Hooreweder, K. Lietaert, B. Pouran, and A. A. Zadpoor, "Effects of applied stress ratio on the fatigue behavior of additively manufactured porous biomaterials under compressive loading," Journal of the Mechanical Behavior of Biomedical Materials, vol. 70, pp. 716, 2017.

[18] T. Stanković, J. Mueller, P. Egan, and K. Shea, “A generalized optimality criteria method for optimization of additively manufactured multimaterial lattice structures," Journal of Mechanical Design, vol. 137, no. 11, 2015.

[19] B. Alberts, A. Johnson, J. Lewis, M. Raff, K. Roberts, and P. Walter, Molecular Biology of the Cell, Chapter 4, DNA, Chromosomes and Genomes, Garland, TX, USA, 6th edition, 2014, ISBN 9780815344322.

[20] R. Nuwer, Counting All the DNA on Earth, The New York Times Company, New York, NY, USA, 2015, ISSN 0362-4331.

[21] A. Mashaghi and A. Katan, "A physicist's view of DNA," De Physicus, vol. 24e, no. 3, pp. 59-61, 2013, http://arxiv.org/abs/ $1311.2545 \mathrm{v} 1$.

[22] R. N. Irobalieva, J. M. Fogg, D. J. Catanese Jr. et al., "Structural diversity of supercoiled DNA," Nature Communications, vol. 6, no. 1, p. 8440, 2015.

[23] S. G. Gregory, K. F. Barlow, K. E. McLay et al., "The DNA sequence and biological annotation of human chromosome 1," Nature, vol. 441, no. 7091, pp. 315-321, 2006.

[24] N. J. Kenny, E. K. Namigai, F. Marlétaz, J. H. Hui, and S. M. Shimeld, "Draft genome assemblies and predicted microRNA complements of the intertidal lophotrochozoans Patella vulgata (Mollusca, Patellogastropoda) and Spirobranchus (Pomatoceros) lamarcki (Annelida, Serpulida)," Marine Genomics, vol. 24, no. 2, pp. 139-146, 2015.

[25] A. Wagschal, "Genome-wide identification of microRNAs regulating cholesterol and triglyceride homeostasis," Nature Medicine, vol. 21, no. 11, pp. 1290-1297, 2015.

[26] Y. L. Phua, J. Y. Chu, A. K. Marrone, A. J. Bodnar, S. SimsLucas, and J. Ho, "Renal stromal miRNAs are required for normal nephrogenesis and glomerular mesangial survival," Physiological Reports, vol. 3, no. 10, article e12537, 2015.

[27] N. D. Amin, G. Bai, J. R Klug et al., "Loss of motoneuronspecific microRNA-218 causes systemic neuromuscular failure," Science, vol. 350, no. 6267, pp. 1525-1529, 2015.

[28] H. John, MC Donald Biological Statistics, University of Delaware, Sparky House Publishing, Baltimore, MD, USA, 2008. 
[29] M. Babič, Analiza kaljenih materialov s pomočjo fraktalne geometrije, Doctoral dissertation, University of Maribor, Maribor, Slovenia, 2014.

[30] L. Lacasa, B. Luque, F. Ballesteros, J. Luque, and J. C. Nuno, "From time series to complex networks: the visibility graph," Proceedings of the National Academy of Sciences, vol. 105, no. 13, pp. 4972-4975, 2008.

[31] P. Dikeakos, G. Theodoropoulos, S. Rizos, N. Tzanakis, G. Zografos, and M. Gazouli, "Association of the miR$146 \mathrm{aC}>\mathrm{G}$, miR-149T $>\mathrm{C}$, and miR-196a2T $>$ C polymorphisms with gastric cancer risk and survival in the Greek population," Molecular Biology Reports, vol. 41, no. 2, pp. 1075-1080, 2014.

[32] J. Kupcinskas, T. Wex, A. Link et al., "Gene polymorphisms of micrornas in Helicobacter pylori-induced high risk atrophic gastritis and gastric cancer," PLoS One, vol. 9, Article ID e87467, 2014.

[33] M. M. Perry, S. A. Moschos, A. E. Williams, N. J. Shepherd, H. M. Larner-Svensson, and M. A. Lindsay, "Rapid changes in microRNA-146a expression negatively regulate the IL-1betainduced inflammatory response in human lung alveolar epithelial cells," Journal of Immunology, vol. 180, no. 8, pp. 5689-5698, 2008.

[34] L. O. Reis, T. C. Pereira, I. Lopes-Cendes, and U. Ferreira, "MicroRNAs: a new paradigm on molecular urological oncology," Urology, vol. 76, no. 3, pp. 521-527, 2010.

[35] Z. Wang, M. Wei, Y. Ren et al., "miR149 rs71428439 polymorphism and risk of clear cell renal cell carcinoma: a casecontrol study," Tumour Biology, vol. 35, no. 12, pp. 1212712130, 2014.

[36] Z. Hu, J. Liang, Z. Wang et al., "Common genetic variants in pre-microRNAs were associated with increased risk of breast cancer in Chinese women," Human Mutation, vol. 30, no. 1, pp. 79-84, 2009.

[37] A. E. Hoffman, T. Zheng, C. Yi et al., "microRNA miR-196a-2 and breast cancer: a genetic and epigenetic association study and functional analysis," Cancer Research, vol. 69, no. 14, pp. 5970-5977, 2009.

[38] I. Catucci, R. Yang, P. Verderio et al., "Evaluation of SNPs in miR-146a, miR196a2 and miR-499 as low-penetrance alleles in German and Italian familial breast cancer cases," Human Mutation, vol. 31, pp. E1052-E1057, 2010.

[39] Z. M. Dai, H. F. Kang, W. G. Zhang et al., "The associations of single nucleotide polymorphisms in miR196a2, miR-499, and miR-608 with breast cancer susceptibility: a STROBEcompliant observational study," Medicine, vol. 95, article e2826, 2016.

[40] W. J. Wei, Y. L. Wang, D. S. Li et al., "Association study of single nucleotide polymorphisms in mature microRNAs and the risk of thyroid tumor in a Chinese population," Endocrine, vol. 49, no. 2, pp. 436-444, 2015.

[41] L. Jiao, J. Zhang, Y. Dong et al., "Association between miR125 a rs12976445 and survival in breast cancer patients," American Journal of Translational Research, vol. 6, pp. 869875, 2014.

[42] J. Kupcinskas, I. Bruzaite, S. Juzenas et al., "Lack of association between miR-27a, miR-146a, miR-196a-2, miR-492 and miR608 gene polymorphisms and colorectal cancer," Scientific Reports, vol. 4, no. 1, p. 5993, 2014.

[43] M. Lin, J. Gu, C. Eng et al., "Genetic polymorphisms in microRNA-related genes as predictors of clinical outcomes in colorectal adenocarcinoma patients," Clinical Cancer Research, vol. 18, no. 14, pp. 3982-3991, 2012.
[44] B. M. Ryan, A. C. McClary, N. Valeri et al., "rs4919510 in hsamir-608 is associated with outcome but not risk of colorectal cancer," PloS One, vol. 7, Article ID e36306, 2012.

[45] R. Wang, J. Zhang, Y. Ma et al., "Association study of miR149 rs2292832 and miR608 rs4919510 and the risk of hepatocellular carcinoma in a largescale population," Molecular Medicine Reports, vol. 10, no. 5, pp. 2736-2744, 2014.

[46] P. Zhang, J. Wang, T. Lu et al., "miR-449b rs10061133 and miR-4293 rs12220909 polymorphisms are associated with decreased esophageal squamous cell carcinoma in a Chinese population," Tumour Biology, vol. 36, no. 11, pp. 8789-8795, 2015.

[47] A. Gutierrez-Camino, E. Lopez-Lopez, I. Martin-Guerrero et al., "Noncoding RNA-related polymorphisms in pediatric acute lymphoblastic leukemia susceptibility," Pediatric Research, vol. 75, no. 6, pp. 767-773, 2014.

[48] Z. Li, W. Zhang, M. Wu et al., "Gene expression-based classification and regulatory networks of pediatric acute lymphoblastic leukemia," Blood, vol. 114, no. 20, pp. 44864493, 2009.

[49] L. R. Treviño, W. Yang, D. French et al., "Germline genomic variants associated with childhood acute lymphoblastic leukemia," Nature Genetics, vol. 41, no. 9, pp. 1001-1005, 2009.

[50] D. Michieletto, D. Marenduzzo, and E. Orlandini, "Is the kinetoplast DNA a percolating network of linked rings at its critical point?," Physical Biology, vol. 12, no. 3, article 036001, 2015.

[51] W. De Nooy, A. Mrvar, and V. Batagelj, Exploratory Social Network Analysis with Pajek, Cambridge University Press, Structural Analysis in Social Sciences, New York, NY, USA, 2005. 


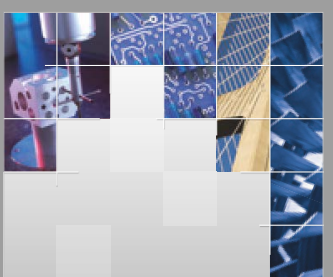

\section{Enfincering}
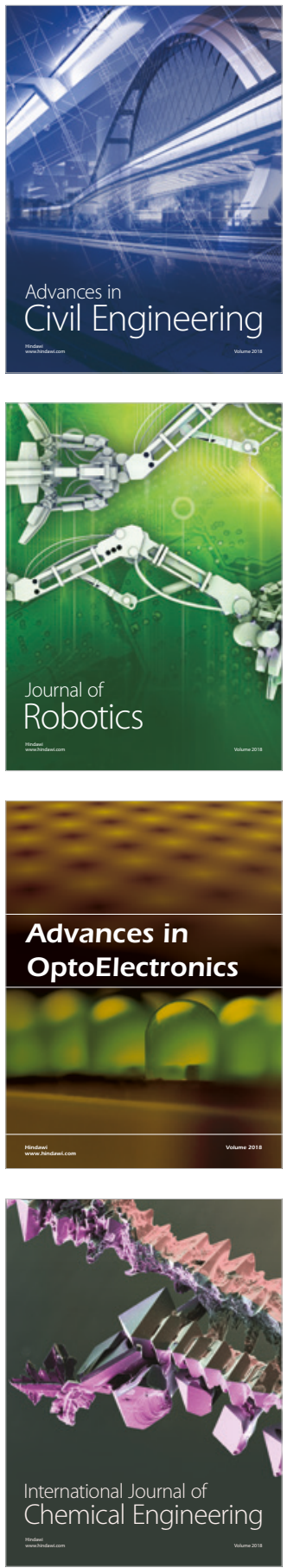

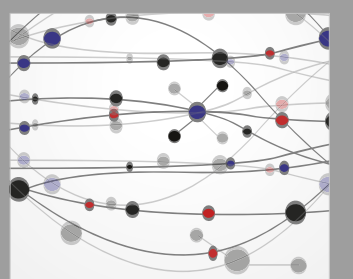

\section{Rotating \\ Machinery}

The Scientific World Journal

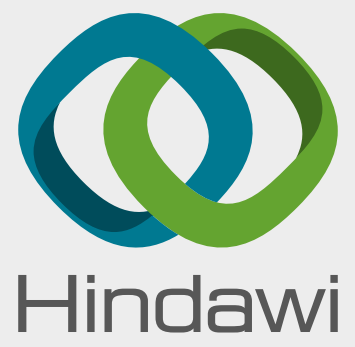

Submit your manuscripts at

www.hindawi.com
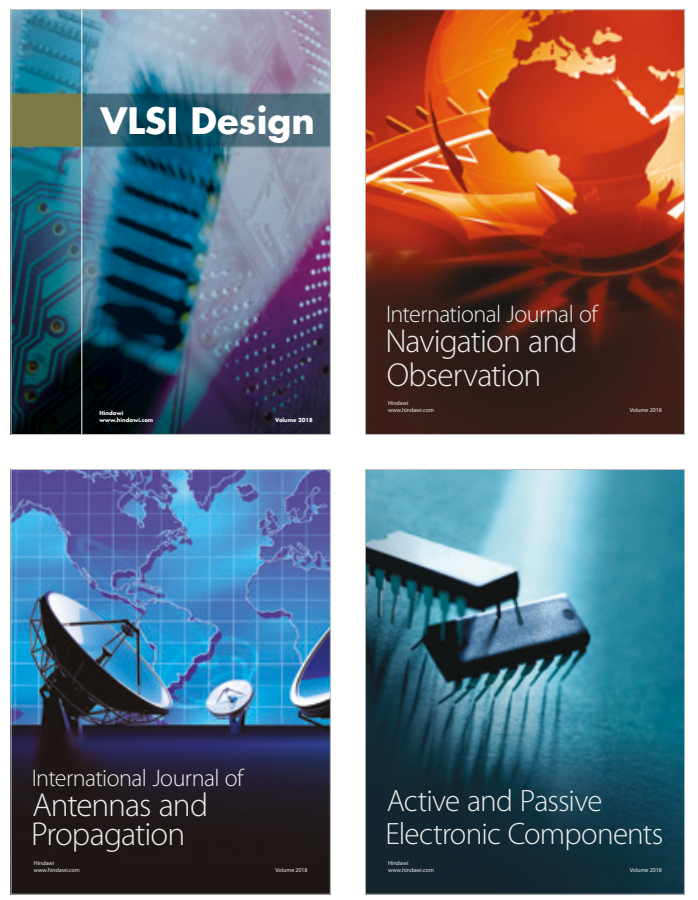
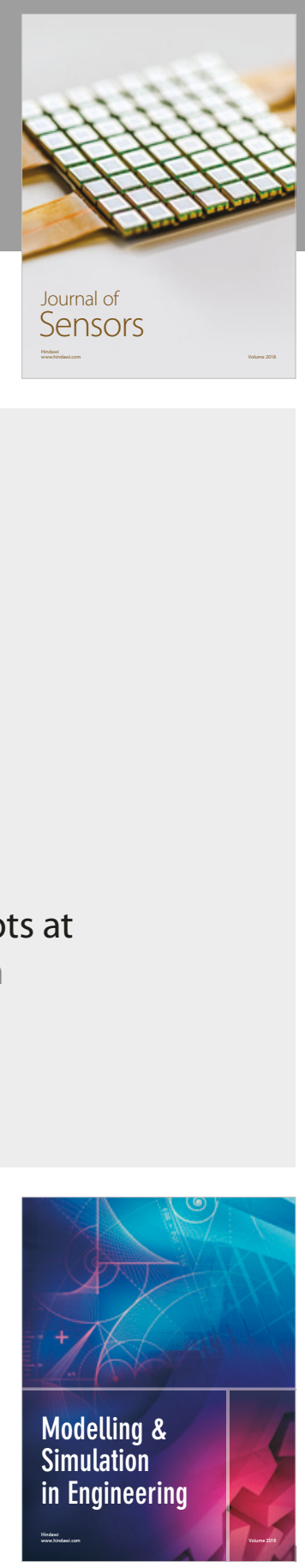

\section{Advances \\ Multimedia}
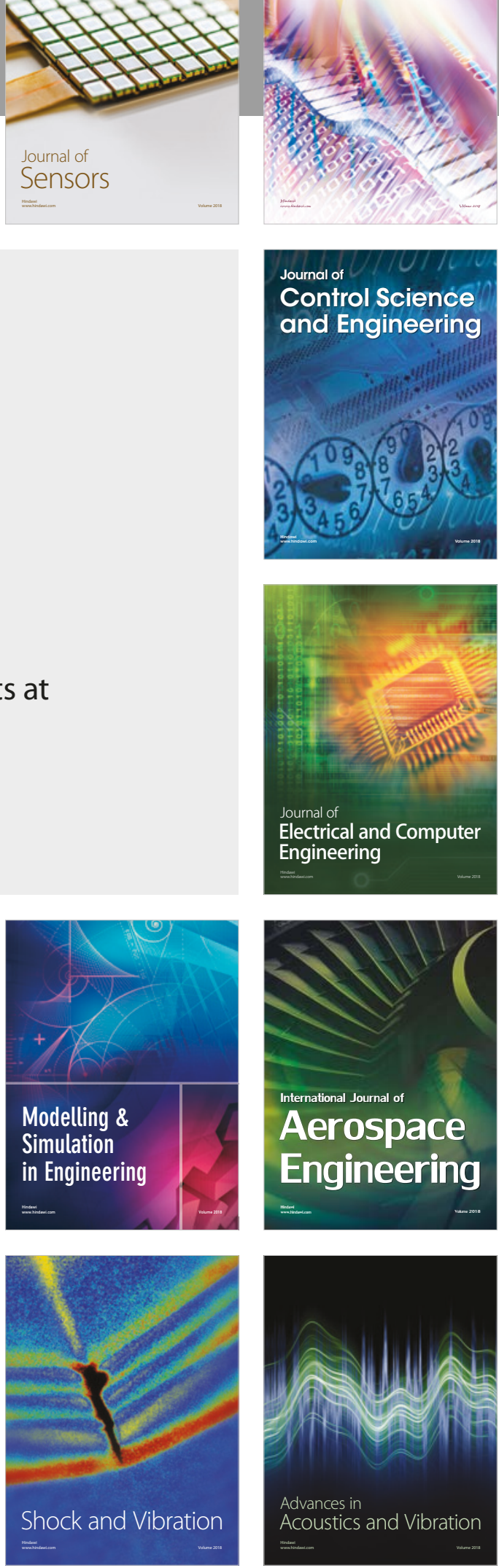